\title{
Zeno's First Paradox of Motion: A Cartesian Perspective
}

\section{Lori Balster}

University of Dayton

Before I introduce some of Descartes' perspective into a discussion of Zeno's First Paradox of Motion, I would first like to restate in general what Zeno's First Paradox of Motion is. Essentially, Zeno says that, in order for motion from point $A$ to point $B$ to occur, one half of that distance must first be traveled. In order for one half of that distance to be traveled, one fourth of that distance must first be traveled, and in order for one fourth to be traveled, one eighth must be traveled, and so on ad infinitum. Since the distance to be traveled can be divided infinitely by the above process, it seemed to Zeno that it would be impossible to ever start traveling a given distance, and that ordinary motion is impossible: hence the Paradox.

Descartes himself took a rather dim view of all those who discussed and tried to solve the First Paradox of Motion. His essential feeling on the matter was that:

We should never enter into arguments about the infinite. Things in which we observe no limits...should be instead regarded as indefinite. Thus we will never be involved in tiresome arguments about the infinite. For since we are finite, it would be absurd for us to determine anything concerning the infinite; for this would be to attempt to limit it and grasp it. ${ }^{1}$

Ironically, his viewpoint actually provides a starting point that I believe leads to a clearer, more satisfying solution to the Paradox than those that have been traditionally proposed. Descartes' objection to arguing about infinity, I believe, actually stems from a very important idea he expresses in his Meditations: the idea that only that which we can perceive "clearly and distinctly" do we know to be true. ${ }^{2}, 3$ The concept of infinity has a certain fuzziness, a ragged edge. We cannot fully comprehend or visualize a series of 
numbers that goes on forever. Therefore, we cannot perceive infinity clearly in mind's eye. Since the nature of infinity is such that it cannot be perceived "clearly and distinctly" by us, the only way in which Zeno's First Paradox of Motion can be discussed in a way acceptable to Descartes is if infinity is removed from the act of motion in the Paradox. I believe that it is not only possible to do this, but that the only way the Paradox can be sufficiently resolved is by removing infinity from it. Descartes' recommendation of only "clear and distinct" perceptions guides the effort at a real solution to the Paradox in this paper. In fact, this paper is an attempt to show that, by taking the Cartesian idea of "clear and distinct" perceptions to heart, the real solving of the Paradox can occur. Through the lens of "clear and distinct," it is eventually shown that the key to unraveling the Paradox lies in the way Zeno has conceived of the act of motion. All other attempts at solving the Paradox accept Zeno's misconception of the act of motion and try to solve the Paradox by manipulating Zeno's misrepresentation of what motion is. These solution attempts are never fully successful, based as they are on Zeno's mistake.

It is important at this point to establish the criteria I will use throughout this paper for evaluating the soundness of a given solution to Zeno's First Paradox of Motion. Each criterion is either directly Cartesian or inspired by Cartesian principles. In order for a solution to the Paradox to be deemed acceptable in the course of this paper, it must:

1) Be clear and distinct. That is, there must be no part of the solution that cannot be clearly perceived with mind's eye.

2) Reconcile our mathematical and physical perceptions. Many attempted solutions of the Paradox address either our mathematical perceptions of motion, as in the infinite geometric series solution, or our physical perceptions of motion, as in Aristotle's solution to the Paradox, but not both. Since both our mathematical and physical perceptions are capable of being clear and distinct enough to reveal truths to us, a truly acceptable, well-rounded solution would address both, and would reconcile them.

3) Not alter concepts such as infinity, time, or distance in an attempt to resolve the Paradox. If the definitions of 
these words are changed, a discussion of clear and distinct perceptions becomes impossible, as the words have lost their usefulness as references to the concepts in question. More importantly, if changed, the words would then mean different things than they would have meant to Zeno, and they could no longer be an appropriate part of the argument at hand. Many attempted solutions to the Paradox are guilty of concept altering in their pursuit of the solution, Benacceraf and Thomson's proposed solutions being but two examples.

Before I begin applying these criteria to specific solutions proposed for Zeno's First Paradox of Motion, I would first like to establish that the need for a better solution still exists, despite the age of the Paradox. Secondly, I intend to show that the solution attempts that do exist do not meet the established criteria. Lastly, I would like to put forth my own solution, and demonstrate that my solution does meet the established criteria. I will proceed through each of these in turn.

Establishing The Need for A Better Solution to the Paradox

Although this particular Paradox was born approximately two thousand years ago, even the most recent attempts at it have not been deemed wholly satisfying. It can be said very truthfully that even in the 21 st century, despite our advanced mathematical apparatus such as infinite geometric series formulae and differential calculus, we have not yet reached a simple consensus on the problem. Attempts continue to be made, of course. But the fact that papers with widely differing viewpoints on the Paradox are still being written suggests that a generally accepted perspective on its resolution is still absent. In the introduction to his collection of essays on the subject, Wesley Salmon notes that Zeno's Paradoxes are sometimes viewed as "mere anachronisms" which would only disturb someone with an inadequate knowledge of calculus. But, as Salmon says, "no evaluation could be further from the truth." 4,5 Salmon is careful to point out that all the widely varying views of the Paradox in his collection come from "mathematically literate 
twentieth-century authors."6 Even the sophisticated mathematics of modern day, many of these authors seem to agree, can't resolve the metaphysical issues of motion. As one such author, J.O. Wisdom, suggests: "We do not eliminate the Paradox until we have seen in what way it fails as a description." R. M. Sainsbury states the matter more specifically in his book Paradoxes when he says, "a full response to Zeno's Racetrack Paradox would require a detailed elaboration and justification of our spatial concepts."

The main reason that solutions that have been proposed for Zeno's First Paradox have not been considered satisfactory by one group or another, it appears, is because in each case the proposed solution for the Paradox contains ambiguities. Each proposed solution contains a part that cannot be seen clearly in mind's eye. A truly satisfying solution-for any problem-has no such parts. Before I present my own approach to this ancient problem, let us briefly revisit the origins of the First Paradox of Motion, examine the solutions to this Paradox that have been proposed so far, and determine how these solutions measure up to the three criteria proposed.

\section{Parmenides}

The Parmenidian approach to space and time is surely the core around which Zeno's First Paradox of Motion is wrapped. The Parmenidian view of time is important to Zeno's First Paradox of Motion because Parmenides' thinking about time left its mark on Zeno, who in Charles Sanders Peirce's words was "the pupil and defender of Parmenides." Bertrand Russell ${ }^{10}$ and others believe that Zeno's primary goal in creating the First Paradox of Motion is to uphold Parmenides' view that our usual conceptions of space and time-our conceptions of space as a procession of discrete distances and of time as a procession of discrete moments-are false. Summarizing the Parmenidian view of time, Ronald Hoy says, "Parmenides finds fault with beliefs about time and argues that time is not real." "In terms of our three criteria, we must reject the view that time is not real, because our physical perception is that time is real and that it does pass (criterion \#2). The reasons for rejecting the view that time is not real, however, are not simple. To 
wit: Parmenides believed that a temporal existence was not possible because, in his view, we exist only in a "durationless now," where we are always in the present in space and time. His belief in the durationless now does not seem entirely unwarranted, as all of us who exist feel that we are experiencing events "now," as opposed to ten minutes ago, a year ago, or a year in the future. Oddly, if time is truly passing, our sense of being in the "now" should not persist-and yet, it does. Perhaps we are the lens through which time passes, but we feel the basic persistence of ourselves. On the other hand, we also feel that we are different today than we were many years ago- having adult thoughts as opposed to childish ones, for instance-suggesting that even our "now" is not untouched by time. Similarly, our personal perception of the "durationless now" begins after our conception, and so our own "durationless now" seems to exist in a specific location in time. Recent evidence also suggests that perhaps our sense of the durationless now is really an illusion. Ilya Farber and Patricia Churchland note in The Cognitive Neurosciences that there is considerable empirical support to suggest that, "when we experience ourselves as having made a conscious decision, what we are in fact getting is a "story" reconstructed from the (external and internal) evidence." 12 In addition, as Brenda Rapp notes in The Handbook of Cognitive Neuropsychology, it is known that the brain does not process experiences instantaneously, so whatever it is we perceive as "now" was several milliseconds ago or more. ${ }^{13}$ In other words, it seems likely that the "durationless now" has some dependence on time.

If it cannot be disputed that time has bearings on the durationless now, the nature of time was nevertheless under dispute until recently. In Parmenides' day, time was often regarded as cyclical. In modern times, the idea that time is linear no longer seems contestable. As G. J. Whitrow explains in The Nature of Time, "The linear view of time as continual progression without cyclical repetition finally prevailed through the influence of the nineteenthcentury biological evolutionists." 14 He continues, "The Darwinian theory of biological evolution... was the decisive factor that caused men to become conscious of the time aspect of the universe." 15 Specifically, because some species proliferate, others die out, and organisms in general become better adapted to their environments 
and more complex, "the chances of retracing the steps of evolution decrease rapidly" the longer the evolutionary process continues. ${ }^{16}$ Parmenides, however, did not have the benefit of modern knowledge. As Hoy says, "Today, it is easy to ignore Parmenides' rejection of time...It is obvious time is real." 17

If it must be conceded that time is real, however, we are not yet finished with Parmenides, as we have not yet shown how time and/or motion can be real, though the issues of the reality of time and motion (i.e. the traveling of a distance) are related. The crux of the issue is the question of how it is possible for a "whole" infinite quantity like time or distance to be divided into discrete units, so that time can be traveled and distance can be crossed. In Zeno's scenario, both must be infinitely divided before a unit of time can pass, or a unit of distance can be traveled. The First Paradox of Motion itself is said to arise largely because the smallestindivisible - unit of distance or time must have fixed dimensions so that the units summed may add up to the total distance or time, and yet each unit must not have fixed dimensions so that it may be divided infinitely, as any distance or time seemingly can be. This results in what is commonly referred to as a plurality: each unit of distance or time must assume the impossible burden of being, in Salmon's words, "both large and small. So small as to have no magnitude, so large as to be infinite."18 Salmon's observation hearkens back to Aristotle's statement about the inscrutableness of any seemingly infinite thing, namely that: "The infinite body must be either (1) compound, or (2) simple; yet neither alternative is possible." ${ }^{\prime 9}$ Hence the basic source of contradiction in motion for Parmenides and his supporters. As Peirce says of Parmenides' pupil, Zeno: "It was an axiom with Zeno that continuity is incomprehensible, and therefore false." ${ }^{20}$ Regardless of our complicated mathematical ruminations on the space/time continuum, however, in the physical world, motion from here to there occurs routinely and with ease. The Parmenidian explanation of motion says that motion only appears simple; our mental perception says that Parmenidian belief cannot be correct because it does not match our experience (a simple, fluid motion). Such a disconnection of explanation and observation would be a violation of criterion \#2, if the Parmenidian position were an attempt at a 
solution to the Paradox, rather than a possible origin of the Paradox itself. When our mathematical explanations match what we actually experience, then, we feel intuitively, we have arrived at the correct view of motion. The Parmenidian approach says, essentially, "this Paradox cannot be resolved," and yet a body resolves the Paradox every time it moves! The disparity between the physical world and the mathematical world is great in the Parmenidian view of time and space. So great, a different approach to space, time, and motion is hoped for. Unfortunately, these different approaches bring with them different problems, as will be reviewed.

\section{Distance and Supertasks}

A possible approach to the solution of the First Paradox of Motion is generated when the question is asked, "what does it mean to travel a distance in Zeno's scenario?" James Thomson designates the task in this case (defined as traveling an infinite distance) as a "supertask," indicating by the label that it is beyond normal reach." He discards the notion that traveling this distance is a "supertask" in the course of things, but because infinity is never removed from his view of the situation, the reasons behind his conclusion are difficult to perceive clearly. He uses a lamp apparatus, for instance, to try to pin down infinity. Proposing a switch which turns the lamp on and off (the "distance" in this case is the distance the switch must be moved from "off" to "on"), he claims that after any number of switchings-even an infinite number-we can be confident of the outcome: the lamp will be on or off. ${ }^{22}$ The finite series conclusion and the "infinite series" conclusion in Thomson's scenario are the same. The similar conclusion of the two seriesas well as some other points Thomson offers up-are ultimately supposed to suggest that "a man who completes a journey completes an infinite number of journeys." ${ }^{23}$ The problem with this solution, of course, is that infinity is not truly being represented. No one could ever finish an infinite series of lamp switches, meaning that Thomson's solution violates criterion \#3. Infinity is a special concept that is useful precisely because the idea it contains-that of a succession of elements without end-is not covered by any other concept. Since Thomson altered his idea of infinity in a 
fundamental way, a way that resulted in his reaching an end of a proposed "infinite" series, he changed what the concept of infinity meant and it no longer referred to the same infinity discussed in the Paradox-hence the criterion \#3 violation. Thomson's lamp scenario also violates criterion \#1, because at the ceiling rate at which the theoretical switching could be occuring, ${ }^{24}$ it is even possible that the lamp would not stay in a state long enough to be called "off" or "on." It may very well occupy a third, intermediate state most of the time: "off-on." We cannot say with confidence what the state of the lamp is, because we cannot completely visualize it. We cannot, therefore, make it comply with criterion \#1. In another attempt to pin down infinity via his conception of distance, Thomson tries to make an analogy between a unit of distance and a lump of chocolate, saying if you divide chocolate long enough you'll go smaller than the molecular level and you no longer have chocolate. ${ }^{25}$ I agree there cannot be a "chocolate atom." Still, distance can be divided in mind's eye without reaching an instant where we can say, "That is not a distance, it is a separate thing which we can call by another name." However, according to some, such as Grünbaum, ${ }^{26}$ distance can be reduced this way-to an entity with no length in any of the three dimensions, i.e. a point. This reduction of a line to a point is, for example, against conventional Euclidean thinking, which says that only lines can comprise larger lines. Grübaum explicitly states the geometric paradox that cannot be avoided in the approach under discussion: if a line consists of points, one should be able to add up these points and arrive at a line length. But points have no length. ${ }^{27}$ Nevertheless, the points must have magnitude in order to be compounded. However, as Owen points out, if a thing has no dimensions its location cannot be fixed, and it does not exist. ${ }^{28}$ Or perhaps, more accurately, since we cannot clearly and distinctly perceive a point (we cannot clearly visualize something with no length), we cannot say that it definitely exists. The point has a "where" to use Aristotle's categories, but not a "position," since it has no magnitude. A collection of points "added" cannot be made into a distance, seeing as how zero dimension, however many times you add it, will not equal a distance. Some have taken this as proof of the Paradox involved in motion. I think it merely shows that distance is a real quantity that does 
exist, and that the point, whose location cannot be defined, does not necessarily exist. We cannot clearly and distinctly perceive a point, as any representation we make of a point, on paper or otherwise, we must make by drawing, or thinking of, a small line. Like Euclidean geometrical figures, the point may also very well have no physical or sensible parallel. Hence, the "point" may not be a valid concept to introduce into ordinary space, which involves distances with fixed location: that is, distances that exist. It seems that we are invoking the observation of Salmon: "Systems of pure mathematics, as such, involve primitive terms which have no physical reference whatever."29 The "physical reference," that is, a way of describing ordinary physical motion in such a way that it does not create internal paradoxes, is what we aim to resolve.

Black, on the other hand, tries to deal with the popular juggling of the physical and mathematical distances by claiming that an infinite mathematical distance does not apply to ordinary distance involved in motion, even though the general consensus is that ordinary distance is infinitely divisible (a violation of criterion \#2). ${ }^{30}$ Wisdom also tries to deal with the math/physical distance split in the same unfortunate way, when he states: "saying Achilles' distance to travel is $1+1 / 2+1 / 4+\ldots$..etc. and saying Achilles' distance is physical is contradictory." 31 Also, "it is impossible," Wisdom says, "to subdivide the distance between [the points] without altering the assigned size of the points." 32 But it should not be impossible for these distances to coexist. Mathematics was invented partly to describe and predict physical reality. The one should illuminate the other; each has that power. So we must turn to yet another approach.

\section{Supertasks and Infinite Motion Machines}

Black and Thomson's notion of "supertasks" contains the idea that the overcoming of the distance by the runner can only be achieved through use of extraordinary methods. In many treatments, these methods involve people or things that can perform infinite tasks, such as the infinite motion machines discussed by Poundstone: the Peano machine and the pi machine. ${ }^{33}$ But such devices can't be satisfactory for solving the Paradox because they 
are-let's face it —not required for ordinary motion (violations of criterion \#2). But when it is believed that ordinary motion involves infinite division, such fantastic apparatus becomes common in attempts at solutions. In addition to the infinity machines, there are other fantastic conceptions, such as Russell's medically impossible example of a person who improves at a task until it takes him no time to do it, ${ }^{34}$ and Benacerraf's genie who shrinks until it allegedly disappears at $1,{ }^{35}$ to name but two. None of these, however, can be clearly perceived, and so they are violations of criterion \#1. As to Russell's, we can't clearly conceive of a person taking no time at all to do a task: The tasks we know have a distinct beginning and end. As to Benacceraf's, at the very moment the genie is supposed to disappear, our otherwise clear vision of the scenario dims. We can't "see" the genie disappearing. We do not know: he might still be there, only just beyond our field of vision. For all we can perceive, he may be hiding like the parameciumin plain view. We surely hope for a better resolution than this.

\section{Time}

Another way of approaching the problem of infinity is to manipulate notions of time (these are of course bound up with attempts at manipulating notions of distance). One attempt is that of Sainsbury ${ }^{36}$ and Black, ${ }^{37}$ who claim that, like a finite distance, a finite block of time consists of an infinite number of subdivisions. Therefore, according to this reasoning, a person completes an infinite number of journeys when he completes one finite journey. The problem with this view is that a body does not experience time in this way-this fact conflicts with criterion \#2. A body experiences a moment in a moment: it never finds itself "unable to finish" a moment. The basic idea of infinity rests on a body's sense that it is doing something forever. If that sense is lost, true infinity is no longer being discussed (criterion \#3). Yet if time is real, then motion must be real. And if motion is real...the problem of resolving the apparent Paradox of Zeno's is still important and infinity must be subdued somehow. It seems that the concept of infinity is not preserved in Sainsbury and Black's approach. A real solution to this Paradox should integrate all the pertinent facts known about 
motion. Sainsbury and Black's perspective will hopefully be superseded by an explanation for which the common notion of infinity is preserved. Grünbaum mentions that clock measures are "finitely additive" like distances, ${ }^{38}$ however, like distances, if time is to be accounted for here, the idea of infinity must be invoked but the numerable/denumerable disparity still exists.

Salmon ${ }^{39}$ points out that before the 19 th century, there was doubt about whether a function could "move" to reach its limit. Today, the limit is simply regarded as a number, not a destination, and there is no mystery about reaching it. However, since the drawing of the function is merely a representation of the purely mathematical and is not meant to be an instance of physical motion, this modern view of the function is not terribly important to our concerns with Zeno. There are no "time-consuming" instants in the paper world of the function. The problem with physical motion is that the instants and pieces of time, already popularly defined to be infinite in number, must occur one at a time, hence the usual conundrum, as there is no infinite amount of time for the motion to occur in. Grünbaum's peculiar concept of "durationless" instants is introduced to solve this problem, ${ }^{40}$ but it does not follow criterion \#2-we cannot say with confidence that something like a durationless instant has any real physical meaning - as Owen says, a truly durationless instant does not exist. (The idea of "durationless instant" must be distinguished from Parmenides' idea of "durationless now," which refers to a being's seemingly transcendental sense of being in the "now," which, nevertheless, must exist in time, as the instant the being has experienced has traveled from the future, to the present, to the past before a being has even perceived it, as discussed previously.) Concepts like the "durationless instant" fail to make infinity, not to mention ordinary motion, any less murky. In fact, they add to the general lack of clarity surrounding the concept, since we can't visualize a "durationless instant" clearly (criterion \#1). 


\section{Grappling With Infinity}

Black $^{41}$ makes the claim that only a finite space and time are needed for the runner to make an infinite series of steps. Of course, this doesn't preserve the usual concept of infinity. We cannot see clearly, visualize with mind's eye, how an infinite progression of steps might be contained in a finite one. As was previously mentioned, Black also claims that there is a distinction between numbers and physical distances. ${ }^{42}$ Here, Black seems to be violating criterion \#2. However, the connection between numbers and physical distances should exist, as Zeno was nevertheless capable of stating his First Paradox in terms of physical space. Another attempt at neutralizing infinity was Grünbaum's, wherein he reports a particular proof strategy - originating with Cantor-which states that two infinite collections are equinumerous if each member of one can be paired with another. ${ }^{43}$ This strategy is supposed to allow us to evaluate the size of infinite series, making it possible for us to gauge if one such series is bigger or smaller than another. But, if we are using the usual definition of infinity, both infinite series are equally inscrutable. Criterion \#1 is again a problem. Harold Lee, although not finding the satisfying solution which is craved, at least properly acknowledges the problem, hence providing the first step to an appropriate resolution: "Mathematical techniques are adequate to deal with the situations of which [Zeno's] paradoxes treat, but philosophers have hastened to argue that the techniques are merely elaborate ways of avoiding the rational problem Zeno raises. The philosophers are correct." $44 \mathrm{He}$ continues, "Zeno's analysis, however, divides space and time...and this division has no last term. But actual motion has a point of arriving, a last term."45 He then tries to neutralize infinity by claiming that Zeno's use of a certain "type" of number is not appropriate for the racetrack scenario: "The series of real numbers is a linear continuum, and every rational has a "place" in the series, but the reals do not "include" the rationals in a literal sense, for the rationals and the reals are not defined in the same way." ${ }^{46}$ Rational numbers are those which can be expressed as a whole number or whole number fraction, whereas real numbers can be rational or irrational numbers (irrational numbers being inexpressible as whole numbers or whole 
number fractions, an example being the square root of 3 ). The real series does not "include" the rationals or irrationals in the sense that the linear continuum of real numbers proceeds from, say, 1 to 4, without the explicit acknowledgement of all of the exact numbers in between (pi, for example, cannot be exactly defined, but this does not mean that the real number series cannot move to 3.142 and beyond). He says further that: "the series of rational numbers is a dense series; that is, between every two elements there is another element. ${ }^{\text {477 }}$ However, real distances seem broad enough in definition to encompass both types of numbers. As Aristotle points out, distances are "infinitely divisible," leaving room for both real and rational numbers. Even if we accept the notion that these two types of numbers are truly different, we still come no closer to describing infinity and motion clearly. But Lee has another approach, wherein he tries subdue the infinity in the Paradox by claiming there are two different kinds of infinity: "In terms of an analysis based on the Cantor-Dedekind theories, the first two of Zeno's paradoxes stem from the failure to discriminate between a denumerable infinity composed of discrete elements and a not denumerable infinity composed of continuous elements." 48 Of course, the essential idea of infinity rests on the similarity of these two infinities: each remains inscrutable in mind's eye (a violation of criterion \#1). Nevertheless, Lee attempts to make infinity more manageable by altering its usual definition (against criterion \#3). On a gentler note, Lee concludes that, "Zeno's analysis is not incorrect in the sense of being totally wrong. It is incorrect only in the sense of being incomplete." Lee believes that Zeno is merely using the wrong model, that of the "pseudo-continuum." "This is not to say that continuity is unanalysable, but only that the adequate analysis is not made in terms of discrete parts." 50 And Lee, inspired by Dedekind, says, "the best way...of dealing with a continuum is...not to interpret a continuum as a synthesis of separately given elements or parts." 51 But this continuum must contain discrete elements, since we can travel and speak about one stretch of distance, such as three yards, an inch, or an angstrom, while excluding another on each side of that distance. So, we must hope for another approach to the Paradox. 
Leibniz

Leibniz, too, grappled with the idea of infinity without complete success (an outcome Descartes would have viewed as predictable). He seemed to think, however, that we intuitively understand what infinity means, even though we cannot perceive it clearly and distinctly. As he says, through the role of Ariste in his fictional Conversation of Philarète and Ariste, "The mind does not see the infinite in the sense that it measures the infinite by its thought. However, it is not enough that the end not be in sight, for the mind could hope to find this end; but the mind understands that there is no such end. This is how geometers see...the asymptotic lines of the hyperbola, which they know can never intersect it, though they approach it without end." 52 Philarète (who plays the part of Leibniz in the conversation) responds, "This way of knowing the infinite is certain and incontestable... But though we can conclude from it that there is no ultimate finite whole, it still does not follow that we see a complete infinite thing." ${ }^{33}$ Leibniz agrees with Descartes, therefore, that something infinite cannot be perceived clearly. Leibniz's reasons for believing that infinity cannot be clearly perceived are different, however. Descartes thought that because of our finite nature we could not really grasp infinity; Leibniz thought that God-in his infinite wisdom - was able to pass on to us finite beings enough of a sense of infinity to know its essential properties. ${ }^{54}$ The fact that no infinite line could be clearly represented could also be shown with our finite, human, mathematical knowledge. As Catherine Wilson notes in her Leibniz's metaphysics: A historical and comparative study, Leibniz employed a demonstration where he drew a line, then drew a diagonal line that intersected the first. Lines could be dropped from points on the original line to points on its diagonal, but clearly the diagonal was always longer. How then, could both lines contain the same (infinite) number of points? As Wilson summarizes the results of this demonstration: "A continuous line, Leibniz recognized, could not be generated from infinitesimals, if these were represented as extensionless points." ${ }^{55}$ Wilson continues, "One might conclude that the composition of the mathematical continuum was an insoluble problem, but allow that the calculus was 
nevertheless a success in operation... Outside the realm of pure mathematics, however, this instrumentalist approach was not very satisfactory." She continues, "Certain metaphysical problems appear to presuppose a solution to the composition problem, notably the problems of change and motion... This puzzle is then perceived to lead rapidly into Zeno's paradoxes and so into the labyrinth of the continuum." 57 Leibniz also felt that only part of motion was real, the part which is force or power. As Wilson reports, this view of motion is something Leibniz believed he had recognized about the true nature of motion that Zeno did not recognize. ${ }^{58}$ Of course, our own mental perceptions of ordinary motion counteract the idea that motion isn't completely real (criterion \#2).

\section{The Geometric Series Solution}

Before I propose my own take on this rather durable conundrum, I would like to dispel whatever part of the myth which might still remain in the reader's mind that the "sum of the geometric series" solution is satisfactory, as Benson Mates ${ }^{59}$ and others suggested some time ago. The idea of the geometric series solution is that the distance a runner travels can ultimately be described as the sum of an infinite geometric series where $S=1+x+x^{2} \ldots=1 /$ $(1-x),|x|<1$. In this expression, " $S$ " is the total distance the runner hopes to travel, which equals 2 . The variable " $x$," the geometric ratio, equals $1 / 2$. Mathematically, Zeno's scenario fits neatly, and the sum is reached. This solution, however, is not as tidy as it appears. In order for the geometric series solution to be appropriate, the series must have a common geometric ratio. ${ }^{60}$ In this case, Zeno gives it as $1 / 2$. Nevertheless, the concept of the Paradox is completely preserved even if the ratio isn't the same for each imaginary division. If, rather than taking the original distance, dividing it in half, then dividing the resulting distance in half, etc., we divided the original distance in half, then the next distance into fourths, then the next distance into thirds, etc., the same problem of infinite divisibility would arise. And yet, with the common ratio absent, the geometrical series solution could no longer apply. It is possible, of course, for us to be charitable and say that this solution works mathematically (for indeed there is a situation where it does: 
$x=1 / 2$ ), and to bring the proposed solution to the next level, at which we try to use it to describe real, physical motion. But if we do this, the solution rends itself again-it would be impossible to divide any physical distance exactly in half, and we are once again confronting the previous scenario where we do not have a common geometric ratio, $x$. Even if we still accept the geometric series solution at this point, we must nevertheless recognize that the solution is vague at some points which are very important to real motion: it says nothing about time-i.e., how long it would take for an infinite series to converge-and it says nothing about whether the series actually reaches its limit, or whether it simply gets closer and closer to the limit - the actual reaching of the limit being a mathematical assumption. This lack of clarity is important for the reason cited by Wittgenstein; it adds up to a certain intellectual shortcoming which confounds our attempts to see the matter of infinity in a clear light: "Queer: when one takes [an infinite series] as a matter of course...it loses its whole paradoxical aspect...it is as if I were to be told: Don't worry, this series goes on without ever stopping. We are as it were excused the labor of thinking of an end."61 We simply can't completely visualize the convergence of an infinite series, so we really don't know that it does reach its limit, and the geometric series solution must then contain a violation of criterion \#1. It may also contain a violation of criterion \#3, for if an infinite series were truly represented as infinite, it would not end. Lastly, even if we still believe in the infinite geometric series solution at this point, there is still the question of whether this series applies to real motion. Is real motion a converging infinite series, as Zeno represents it-meaning that it contains the problem of an infinite quantity converging in a finite time - or is it more like a divergent series, which is has no pre-determinable limit? I believe it is the latter, and my reasons for this appear in my own solution to the Paradox. Aristotle also had a few choice words to say about this very matter.

\section{Aristotle}

Aristotle made some rather useful observations about Zeno's First Paradox. Many solutions proposed by authors in this paper 
owe their origins to Aristotle's way of thinking. Unfortunately, Aristotle also attempted to explain Zeno's First Paradox of Motion without removing infinity from it first. As Sir Ross sums up Aristotle's beliefs concerning the Paradox, "The essence of his reply [to the First Paradox of Motion] is that, while it is impossible to traverse an infinite space in a finite time, it is possible to traverse an infinitely divisible space in a finite time, since a finite time is itself infinitely divisible." ${ }^{2}$ I suppose there can be no disagreement with this basic assertion, except that we experience neither the infinite divisibility of time nor infinite divisibility of distance in ordinary motion (criterion \#2). Aristotle himself seemed to sense this, as he says in Physics that each distance seems to contain an infinite number of segments, but that the infinite number of these segments is not an "essential" characteristic of the distance, but rather an "accidental" one. ${ }^{63}$ However, Aristotle does not make it entirely clear how this result can follow. If the infinite nature of a distance (i.e. the fact that a distance can undergo division infinitely) is a characteristic of it, how does this characteristic get demoted from "essential" to "accidental"? Perhaps it is because Aristotle doesn't believe Zeno's assumption that we must divide distances in order to cross them. Aristotle makes the promising observation that "though what is continuous contains an infinite number of halves, they are not actual but potential halves. If the halves are made actual, we shall get not a continuous but an intermittent motion." $\mathrm{He}$ continues, "Therefore to the question whether it is possible to pass through an infinite number of units either of time or of distance we must reply that in a sense it is and in a sense it is not. If the units are actual, it is not possible: if they are potential, it is possible." ${ }^{\text {"Is }}$ In other words, if I travel a distance, and I then try to divide the distance in half, then divide that distance in half, etc., it will seem as though the distance has an infinite property and that it would never have been possible to start traveling the distance. However, if I say that I cannot divide a distance that I have traveled until I have already traveled it (that is, until the "potential" or untraveled distance becomes "actual" because I have traveled it), I have removed the entire problem of the infinite divisibility of the distance - the problem of "starting" the motion. I think Aristotle's observation here is very important. Aristotle, however, did not seem 
to think said observation was very important, as he does not bother to explain it in detail. And, not only does he fail to emphasize it in the Physics, he continues on after the observation into a discourse on "becoming," which really is solvable using the same, simple observation. That is, if we refuse to recognize the final form of the object until it has reached that final form, we cannot enter into a debate about at what moment in its transformation it has reached that final form. I, on the other hand, think that Aristotle's "potential" versus "actual" distance observation is very important, and in fact I use it as a step in my clear and distinct solution, which follows.

\section{A Clear and Distinct Solution}

To arrive at a clear and distinct solution to the First Paradox of Motion, we must first begin in a position of doubt. Not in a position of fundamental doubt, such as that adopted by Descartes at the beginning of the Meditations, but in a position of doubt where we accept that one thing is true, the clear and distinct perceptions of our minds, and nothing else.

Now that we are in a position where we are no longer assuming anything about the First Paradox, let us begin at its most obvious part: the language in which it is couched. We must tum our attention to the fact that the very phrasing of Zeno's Racetrack Paradox seems to have caused some difficulties which have mislead thinkers over the centuries in their attempts at valid solutions. The $x=1 / 2$ of the geometric solution is but the first example where the description of the Paradox causes obstacles to reaching the heart of the matter. Of course, Zeno is not necessarily to blame-all our sources of the Paradox are second-hand fragments at best.

But let us work with what we have. The phrasing attributed to Zeno says that, before a runner can traverse the distance of the racetrack, he must traverse $1 / 2$ the distance, before he can do that, he must traverse $1 / 2$ of $1 / 2$ the distance, etc. Yet this is only an attempt at describing a process of infinite division by $1 / 2$. We know by our own clear and distinct perceptions of motion that this infinite division is not an accurate phrasing of the actual situation encountered by a moving body. In fact, for such a body, the actual geometric series is exactly backward. Thomson has already pointed 
out that the correct sequence order for the distance in Zeno's paradox is in the direction of 0 to $1 / 2$ to 1 , rather than supporting the usual view that it is 1 to $1 / 2$ to 0 , but he fails to make anything pivotal of it. ${ }^{66}$ However, something pivotal does result from this view, as I will show.

Zeno begins his series at the completed distance. An actual body at the start of movement is at zero. The body adds $1 / 8$ to its distance traveled, then $1 / 4$, then $1 / 2$, etc., then ultimately a total distance of some kind (usually referred to as whole number, such as 1 or 2) is reached. Zeno divides the total distance in his series by $1 / 2$, then $1 / 4$, then $1 / 8$, etc., so rather than gaining distance, his series is always losing it: the exact opposite of what a moving body does. As was previously stated, Aristotle portrays the series Zeno pictures as infinite, but that is only because Zeno's visualization of it proceeds in the direction of decrease.

Actual Situation
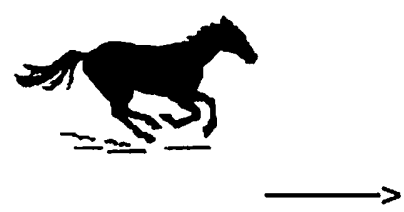

start

\section{Zeno's Visualization}

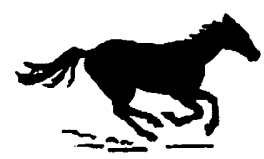

$<$

direction of

finish

infinite series/

direction of

motion

Nevertheless, Zeno proceeds to divide the total distance by a series of halves, never running out of distance that can be halved, and therefore claims that, since these distances can never be halved to completion, the runner can never get started. Hence the Paradox. All agree that Zeno has failed to describe the simple, fluid motion that we physically experience. But all do not agree as to why his idea of motion fails to coincide with natural motion. I believe that his idea of motion deviated from real motion as soon as he visualized the situation. The problem is not mathematical, quantummechanical, or Parmenidian. The problem is the picture in his head. 
I believe that when we combine Aristotle's observations of the "accidental" and "essential" properties of the distance to be traveled, Aristotle's distinction of "potential" and "actual" distances, Thomson's observation of the real direction of the sequence (in the direction of 0 to 1 ), and Bergson's comment, where he notes that "the possibility of applying the movement (of the runner) to the line traversed exists only for an observer who...tries to reconstruct the real movement," ${ }^{167}$ we are on our way to finding the truth about Zeno's First Paradox.

I take this view a step further, claiming that the "line traversed" doesn't exist in Zeno's setup of the Paradox. There is no "distance traveled" until the runner has actually traveled it. Because this distance only arises after the runner's movement, it does not have a fixed value. It can be assigned any value at all, until the runner has completed it (the runner has ceased motion), at which point it has a specific value. Before the runner has traveled, the "distance traveled" is mere speculation. To put this idea in other terms, the distance traveled cannot be expressed as a fixed sum, such as 1 or 2 , or in the infinite geometric series solution, $S$, before the runner has moved it. Before the distance has been traveled, it is unbounded. It cannot be expressed as a finite length until it has been traveled, at which point the motion has already occurred, and there is no "Paradox of Motion" to be had. If the series were infinite, as it seems to be proceeding in the direction of infinite increase rather than in the direction of infinite decrease in Zeno's scenario, we must admit that it has no bound, like any other infinite increasing series. When the motion ceases, however, the series is halted, and a limit is reached-the series is no longer infinite, as it describes real motion, which has a point of arriving. We have eliminated the problem of the convergence of an infinite series. And here we come to Zeno's basic error in describing this situation, and the general problem inherent in anyone's solution that relies on Zeno's description of the runner and the distance.

We see, then, that this approach to the Paradox is the only kind that does not contain the usual thorniness. It is clear and distinct, which fulfills criterion \#1. It has no parts that cannot be perceived by mind's eye. It reconciles our physical and mathematical perceptions, which fulfills criterion \#2. It also does not change the 
usual definition of infinity, time, distance, or any such concepts in an attempt at resolving the Paradox, which fulfills criterion \#3. All the criteria, therefore, have been met.

This Paradox, I think, shows us how important visualization is in problem solving. The one part of Zeno's perspective that everyone used when analyzing Zeno's First Paradox was Zeno's own visualization of motion. Everyone trusted that he had at least framed the basic situation correctly. Since Zeno's visualization was incorrect, no one could come up with a clear solution to the problem. And so the problem remained. It remained long enough to exasperate Descartes. It remained long enough to cause many mathematicians and philosophers pause. But now, hopefully, the troublesome nature of this Paradox has been fully answered.

\section{Notes}

${ }^{1}$ René Descartes, The Philosophical Writings of Descartes: Volume I, trans.John Cottingham, Robert Stoothoff, Dugald Murdoch (Cambridge: Cambridge UP, 1987) pp. 201-2.

${ }^{2}$ René Descartes, Meditations on First Philosophy In Which The Existence of God And The Distinction of the Soul from the Body Are Demonstrated, trans. Donald A. Cress (Indianapolis: Hackett, 1979) p. 35.

${ }^{3}$ The expression "clearly and distinctly" refers to that which we can perceive without having any doubts, or any ambiguity about the perception. If wax melts in our hand, to use Descartes' example from the Second Meditation, pp. 30-1 in the work referenced above, we "clearly and distinctly" perceive the transformation of the wax, since the experience cannot be doubted, it is so directly perceived by the mind.

${ }^{4}$ Wesley C. Salmon, introduction, Zeno's Paradoxes, ed. Wesley C. Salmon (Indianapolis: Bobs-Merrill, 1970) p. 5.

${ }^{5}$ William Poundstone reports the general attitude towards Zeno's Paradoxes—which Salmon rejects—when he states, "To modern minds, some of Zeno's arguments are less compelling. Zeno is apt to come off as a mathematical crank who never got the hang of infinite series." Labyrinths of Reason: Paradox, Puzzles, and the Frailty of Knowledge (New York: Doubleday, 1988) p. 145.

6 Salmon, p.5. 
7 J. O. Wisdom, “Achilles on a Physical Racecourse," Zeno's Paradoxes, p. 87.

${ }^{8}$ R. M. Sainsbury, Paradoxes, (Cambridge: Cambridge UP, 1988) p. 21.

${ }^{9}$ Charles Sanders Pierce, "Lowell Lecture II, September-October 1866," Writings of Charles S. Peirce: A Chronological Edition, ed. Max H. Fisch (Bloomington: Indiana UP, 1982) pp. 390-1.

${ }^{10}$ Bertrand Russell, "The Problem of Infinity Considered Historically," Zeno's Paradoxes, pp. 54-5.

"Ronald C. Hoy, "Parmenides' Complete Rejection of Time," The Journal of Philosophy 91 (1994): 573.

${ }^{12}$ Ilya B. Farber and Patricia S. Churchland, "Consciousness and the Neurosciences: Philosophical and Theoretical Issues," The Cognitive Neurosciences, ed. Michael S. Gazzaniga (Cambridge: MIT Press, 1995) p. 1299.

${ }^{13}$ Brenda Rapp, The Handbook of Cognitive Neuropsychology: What Deficits Reveal About the Human Mind (Philadelphia: Taylor and Francis, 2001) p. 152.

${ }^{14}$ G. J. Whitrow, The Nature of Time (New York: Holt, Rhinehart \& Winston, 1972) p. 25.

is Ibid., p. 149.

${ }^{16}$ Ibid., ibid.

${ }^{17}$ Hoy, p. 573.

${ }^{18}$ Salmon, p. 13.

${ }^{19}$ Physics,III,5,204b11-14.

${ }^{20}$ Peirce, pp. 390-1.

${ }^{21}$ James Thomson, "Tasks and Super-Tasks," Zeno's Paradoxes, p. 90.

${ }^{22}$ Ibid.; p. 94.

${ }^{23}$ Ibid.; p. 100.

${ }^{24}$ The speed of light

${ }^{25}$ Thomson, pp. 90-1.

${ }^{26}$ Adolf Grünbaum, "Zeno's Metrical Paradox of Extension," Zeno's Paradoxes, pp. 180-1.

${ }^{27}$ Adolf Grünbaum, "Modern Science and Refutation of the Paradoxes of Zeno," Zeno's Paradoxes, p. 165.

${ }^{28}$ G.E.L. Owen, "Zeno and the Mathematicians," Zeno's Paradoxes, p. 155.

${ }^{29}$ Salmon, p. 33.

${ }^{30}$ Maxwell Black, "Achilles and the Tortoise,"Zeno's Paradoxes, p. 81.

${ }^{31}$ Wisdom, p. 86. 
32 Ibid.; p. 87.

${ }^{33}$ Poundstone, pp. 147-9.

${ }^{34}$ Sainsbury, p. 14.

${ }^{35}$ Paul Benacerraf, "Tasks, Super-Tasks, and the Modern Eleatics," Zeno's Paradoxes, p. 117.

${ }^{36}$ Sainsbury, p. 14.

${ }^{37}$ Black, p. 69.

${ }^{38}$ Grünbaum, "Modern Science and Zeno's Paradoxes of Motion," Zeno's Paradoxes, p. 206.

${ }^{39}$ Salmon, p. 22.

${ }^{40}$ Grünbaum, "Modern Science and Zeno's Paradoxes of Motion," p. 209.

${ }^{41}$ Black, p. 69.

${ }^{42}$ Ibid.; p. 80.

${ }^{43}$ Grünbaum, "Modern Science and Refutation of the Paradoxes of Zeno," p. 167.

${ }^{44}$ Harold N. Lee, "Are Zeno's Paradoxes Based on a Mistake?" Mind 74 (1965): p. 563.

${ }^{45}$ Ibid.; p. 565.

${ }^{46}$ Ibid.; p. 566.

${ }^{47}$ Ibid.; p. 567.

${ }^{48}$ Ibid.; p. 569.

${ }^{49}$ Ibid.; p. 567.

${ }^{50}$ Ibid.; p. 569.

${ }^{51}$ Ibid.; Ibid.

52 Gottfried Leibniz, "Conversation of Philarète and Ariste," Philosophical Essays, ed. and trans. Roger Ariew and Daniel Garber (Indianapolis: Hackett, 1989) p. 267.

${ }^{53}$ Ibid., p. 267.

${ }^{54}$ Ibid., p. 268

ss Catherine Wilson, Leibniz's metaphysics: A historical and comparative study, (Princeton: Princeton UP, 1989) p. 75.

${ }^{56}$ Ibid., ibid.

${ }^{57}$ Ibid., p. 204.

${ }^{58}$ Ibid., p. 205.

${ }^{59}$ Benson Mates, Skeptical Essays, (Chicago: University of Chicago Press, 1981) p. 7.

${ }^{60}$ Michael Sullivan and Michael Sullivan, "Geometric Sequences; Geometric Series," Algebra and Trigonometry Enhanced with Graphing Utilities, (Saddle River: Prentice Hall, 1996) pp. 857-858.

${ }^{61}$ Ludwig Wittgenstein, Remarks on the Foundation of Mathematics, eds. G.H. von Wright, R. Rhees, G.E.M. Anscombe trans. G.E.M. 
Anscombe (Cambridge: MIT Press, 1983) p. 272.

${ }^{62}$ Sir David Ross, Aristotle, (New York: Routledge, 1996) p. 93.

${ }^{63}$ Physics, VIII,8,263b6-9.

${ }^{64}$ Physics, VIII,8,263a23-30.

${ }^{65}$ Physics, VIII,8,263b3-5.

${ }^{66}$ James Thomson, "Tasks and Super-Tasks," p. 97.

${ }^{67}$ Henri Bergson, "The Cinematographic View of Becoming," Zeno's Paradoxes, p. 65. 